\title{
Computational Analysis of Magnetic Droplet Generation and Manipulation in Microfluidic Devices
}

\author{
Venoos Amiri Roodan', Jenifer Gómez-Pastora², Aditi Verma ${ }^{1}$, Eugenio Bringas², Inmaculada Ortiz ${ }^{2}$, \\ Edward P. Furlani ${ }^{1}$ \\ ${ }^{1}$ Department of Chemical and Biological Engineering, University at Buffalo SUNY \\ Buffalo, New York 14260, USA \\ venoosam@buffalo.edu \\ ${ }^{2}$ Department of Chemical and Biomolecular Engineering, University of Cantabria \\ Avd. los Castros, s/n, Santander, 39005, Spain \\ gomezjp@unican.es
}

\section{Extended Abstract}

Droplet based microfluidics involves the generation and manipulation of discrete volume of fluid (droplets or dispersed phase) in an immiscible (continuous) phase. The interest in this technology had grown dramatically as it holds great potential to provide innovative solutions for numerous applications that range from fast analytical systems of the synthesis of advanced materials to biological assays for living cells [1]. However, to realize the full potential of this technology, precise control of the droplet volumes and reliable manipulation of individual droplets including coalescence, mixing and sorting etc. are needed. In this presentation we discuss magnetic control of droplets.

Magnetic separation has proven a useful and elegant method for manipulating magnetic particles or magnetically labelled biomaterials in microfluidic devices. Magnetic fluids known as ferrofluids are colloidal suspensions in which magnetic nanoparticles (MNP) are dispersed in a carrier fluid. Magnetic forces, provided by and external field source, are commonly used as dominant controlling factors to manipulate the behaviour of these fluids in laminar flow streams [2].

In this work, we present a computational fluid dynamic (CFD) model to investigate the dynamics of oil-based ferrofluid droplets within an aqueous continuous phase. The CFD is performed using the volume-of-fluid (VOF) method as implemented in the commercial program $\boldsymbol{F L O W - 3 D}$ (www.flow3d.com). For droplet generation, microfluidic T-junction is studied. The flow solver was linked to a custom FORTRAN subroutine that calculates the magnetic field numerically due to a rare-earth permanent magnet field source and the corresponding magnetic force exerted on the droplets. The solver solves for magnetic field and magnetic flux density then calculates for magnetization accordingly via Langevin function. The magnetic force on each droplet is calculated using kelvin body force. The Flow-3D helps to demonstrate how the external magnetic force manipulates shape of droplets.

We introduce an optimization study for the continuous processing of droplets under magnetic field generated by a permanent magnet. The magnetic separation and droplet deflection is presented as a function of impact variables and parameters. Parameters such as magnetic location, flow rates of continuous aqueous phase and fluid properties, fluid properties, etc. Our results show that the effect of these factors can be tuned and optimized through various chip designs, depth of the channel, and hydrodynamics of the both oil and aqueous phases. The optimization includes magnetic integration via numerical method and fluidic computational models that accurately describe the droplet generation and motion under different magnetic field and flow conditions. Overall, the model enables better understanding of physical phenomena involved in the continuous droplet processing and suggests an efficient parametric analysis and optimization platform. Furthermore, the results of this study can be used to rational design of microfluidic chip systems.

\section{References}

[1] S.-Y. Teh, R. Lin, L.-H. Hung, and A. P. Lee, "Droplet microfluidics," Lab on a Chip, 10.1039/B715524G vol. 8, no. 2, pp. 198-220, 2008. 
[2] A. Q. Alorabi et al., "On-chip polyelectrolyte coating onto magnetic droplets - towards continuous flow assembly of drug delivery capsules," Lab on a Chip, 10.1039/C7LC00918F vol. 17, no. 22, pp. 3785-3795, 2017. 